\title{
Periodic textures for enhanced current in thin film silicon solar cells
}

Franz-Josef Haug, Thomas Söderström, Oscar Cubero, Vanessa Terrazzoni-Daudrix, Xavier Niquille, Stephanie Perregeaux, and Christophe Ballif

Institute of Microtechnology, University of Neuchatel, Rue A.-L. Breguet 2, Neuchatel, Switzerland

\section{ABSTRACT}

For thin film silicon solar cells it is vital to increase the optical path of light in the absorber because this allows for thinner cells with better stability and higher production throughput. We discuss the effect of periodically textured interfaces for the case of thin film silicon solar cells in n-i-p configuration using embossed plastic substrate which allows us studying the effect of a wide range of random or periodic textures. Due to the moderate thickness of the individual layers the texture is carried into each interface with a high degree of conformity even for the front contact which is the last layer. Solar cells on periodic structures show excellent performance; in a microcrystalline cell on a simple sinusoidal grating we achieved a gain in current density of $30 \%$. Furthermore, the periodicity serves as a useful tool for the study of light management because the underlying phenomena like diffraction or grating coupling to plasma excitations of the metallic back reflector are governed by a relatively low number of well defined parameters like the periodicity and the amplitude of the grating.

\section{INTRODUCTION}

Soon after the first fabrication of an amorphous solar cell by Carlson [1] it was suggested that the utilization of incoming light in the device could be enhanced by light scattering at rough interfaces [2]. Further on, light trapping developed into an essential feature because the adverse effects of light induced degradation are less severe in thin cells [3]. Since then a wealth of structuring methods has been proposed and tested. Most often structuring is achieved in the contact layers during their deposition. For example, pin devices usually employ transparent front contact materials that develop a texture during their deposition on the glass substrate [4-6]. Similarly, the natural textures of polycrystalline metal layers are used for nip devices [7]. Recently, light scattering by the excitation of localized plasma oscillation in metallic nanoparticles has gained considerable interest; enhanced intensities of the electric field in their vicinity has been found useful in organic semiconductor solar cells where poor charge carrier mobility necessitates very thin absorber structures [8-10]. The effect was also reported to increase the photocurrent in silicon thin film solar cells [11]. 
Besides randomly textured interfaces, also periodic structures have been suggested for enhancing the performance [12-14]. Compared to random interfaces, the prediction of some of their properties is possible without undue complications in the mathematical modeling. This makes them a powerful tool towards a better understanding of light trapping in the device, but they could also become an interesting option for production because large area roll to roll processing allows the fabrication of periodic structures with promising gains in performance [15]. In this contribution we present some basic phenomena of light scattering and diffraction at periodically structured interfaces, and we address an important loss mechanism by the excitation of surface plasmon polaritons (SPPs).

\section{EXPERIMENTAL}

The gratings studied in this investigation are produced on a regular basis by a commercial supplier (OVD Kinegram AG, Switzerland). They are fabricated by large area roll-to-roll replication of a grating master into the surface of transparent plastic substrates like PET and PEN. The process is fully compatible with large area requirements of solar cell applications and is applicable not only to gratings but also to a wide range of arbitrary surface structures. In this study we use an exemplary grating which consists of a 1D sinusoidal structure with a period of $890 \mathrm{~nm}$ and an amplitude of $70 \mathrm{~nm}$ (half the peak to valley depth). The textured plastic substrates are covered with sputtered films of silver and zinc oxide without intentional heating (Univex 450B, Leybold). The amorphous silicon was fabricated by plasma enhanced chemical vapour deposition (PECVD) at $70 \mathrm{MHz}$ and a substrate temperature of $190^{\circ} \mathrm{C}$ from a silane/hydrogen mixture with dilution $\left[\mathrm{H}_{2}\right] /\left[\mathrm{SiH}_{4}\right]$ equal to two. Reflection and transmission spectra for the visible and near IR were recorded with non-polarized light in a spectrometer with integrating sphere (Lambda 900, Perkin Elmer), measurements in the mid IR range were carried out with a FTIR setup (Nicolet 8700, Thermo). The spectral measurement of the absorption dips may somewhat limit the resolution compared to an angular scan at fixed wavelength, but it gives access to a wider range of wave vectors in a single measurement.

\section{RESULTS AND DISCUSSION}

\section{$\underline{\text { A: Diffraction phenomena }}$}

Diffraction is a rather old and well studied phenomenon in optics, and a wide variety of approaches with different levels of mathematical rigor are in use [16]. In this section we present properties of sinusoidal gratings that are well understood within a simple scalar diffraction theory, but we will make use of non-paraxial extensions suggested by Harvey [17]. The angular properties of reflection at a periodic structure are given by the well known grating equation:

$$
n_{1} \sin \theta_{d}-n_{2} \sin \theta_{i}=m \cdot \frac{\lambda}{L}, \quad m=0, \pm 1, \pm 2, \pm 3, \ldots
$$

Here, $\theta_{\mathrm{i}}$ and $\theta_{\mathrm{d}}$ denote the incident angle and the diffracted angle, the sign conventions for the angles in reflection and transmission are illustrated in Figure 1. The refractive indices of the

materials in which the respective beams propagate are denoted by $n_{1}$ and $n_{2}$. The wavelength and 
the periodicity of the grating are denoted by $\lambda$ and $L$, respectively, and the diffraction order is given by the integer number $m$. In case of reflection the refractive indices are obviously equal, but positive diffracted angles $\theta_{\mathrm{d}}$ are measured towards the right of the surface normal (negative mathematical sense). Figure 1 shows the diffraction angles for the case of an $890 \mathrm{~nm}$ reflection grating in air, assuming vertical incidence.

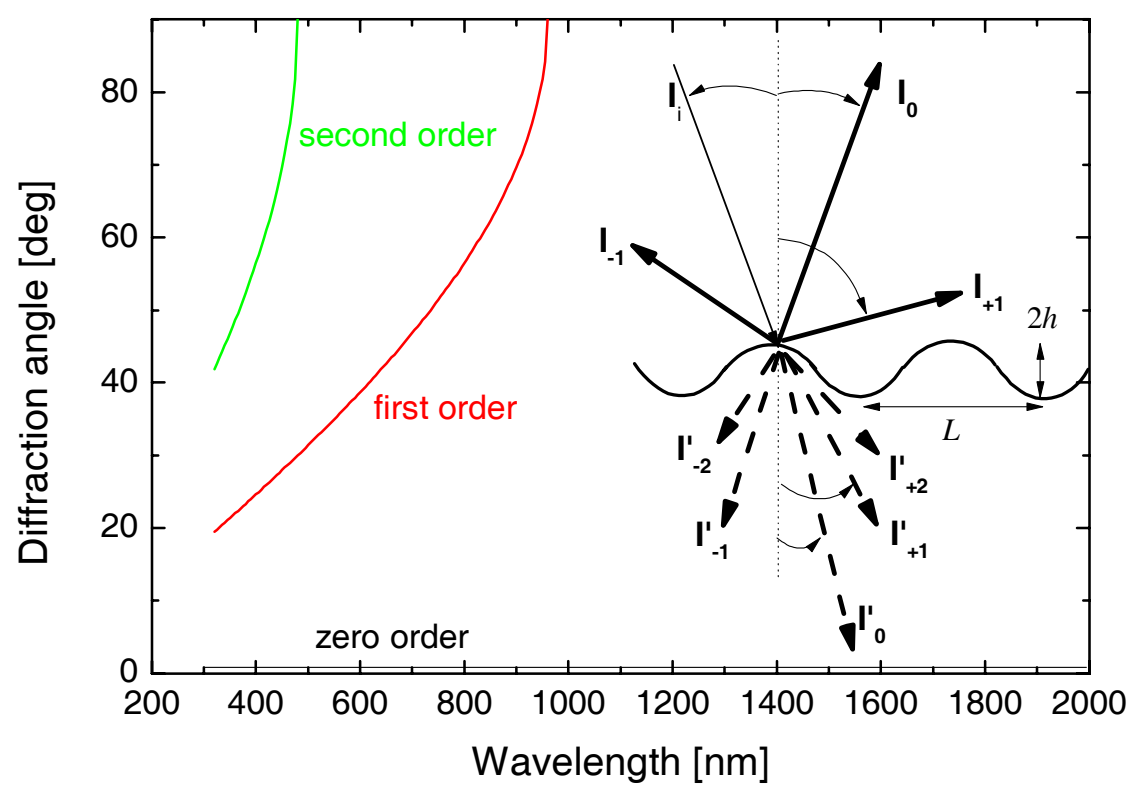

Figure 1: Diffraction angles for a reflection grating with a period of $890 \mathrm{~nm}$ in air. The inset illustrates the propagation of different orders in reflection (full arrows) and transmission (dashed arrows), and the sign convention for the diffraction angles.

For the application of a given grating it is equally important to know how much intensity is diffracted into the different orders. For simple geometries like rectangular, saw tooth and sinusoidal gratings exact solutions do exist. For the latter case the intensity of the $m$-th order is proportional to the square of the $m$-th order Bessel function [16]:

$$
I_{m} \sim J_{m}^{2}\left(\frac{2 \pi h}{\lambda} \cdot\left(\cos \theta_{i}+\cos \theta_{d}\right)\right)
$$

The argument of the Bessel function contains the amplitude $h$ of the grating which is half the peak to valley depth. In a mathematical sense the sum over all Bessel functions is normalized, but physically the situation is different; for example, under perpendicular incidence and wavelengths longer than the grating period, the only possible solutions of eq (1) is specular diffraction into the zero order beam. Diffraction into first (and higher) order modes is forbidden. Figure 2 shows exactly this situation for the case of reflection from the $890 \mathrm{~nm}$ grating. In the wavelength range between 1000 and $2000 \mathrm{~nm}$ we find that all intensity (except for some minor absorption) is reflected into the specular beam. The diffuse reflection which measures light that is diffracted into non-zero order is close to zero in this range. 


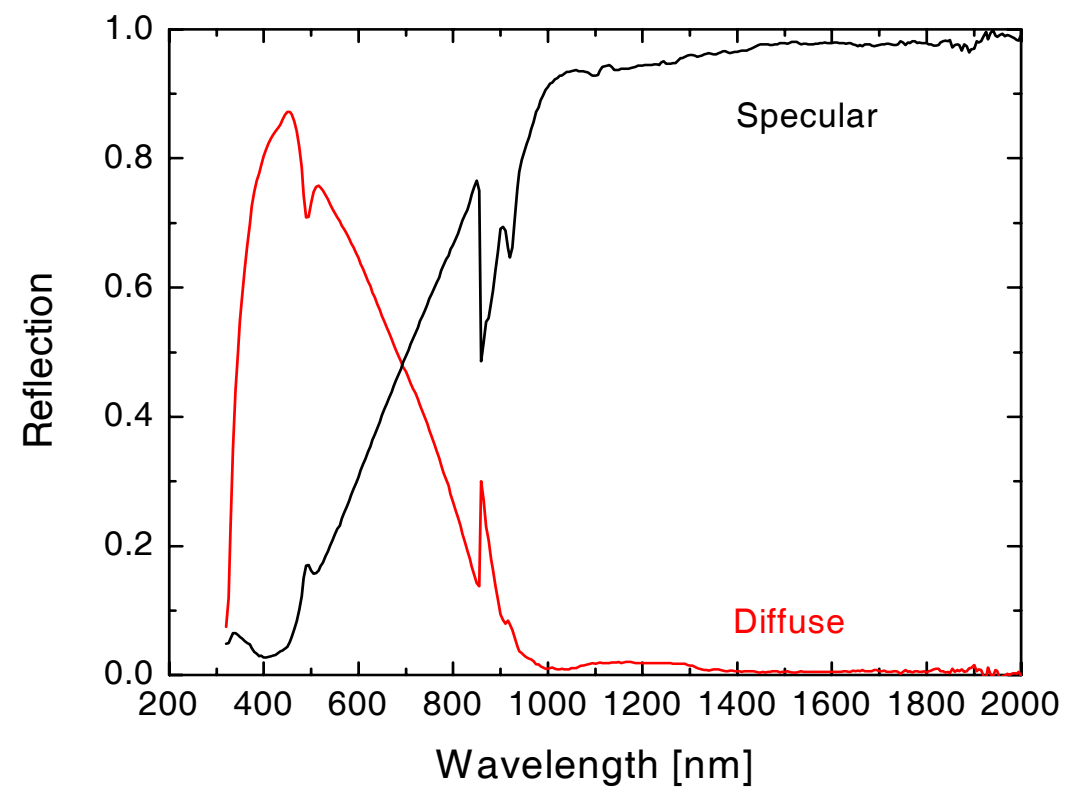

Figure 2: Specular and diffuse reflection of the sinusoidal grating coated with silver.

However, different from the measurement, Figure 3 shows that the intensity predicted by eq. (2) would be lower than one. The situation is ameliorated by a normalization procedure of Harvey [17]; he proposes that the intensities according to eq. (2) should be divided by the sum over the intensities of all propagating modes. Assuming again vertical incidence, Figure 3 shows that this procedure yields a specular intensity equal to one for all wavelengths longer than the grating period because there is only propagating mode, the zero order.

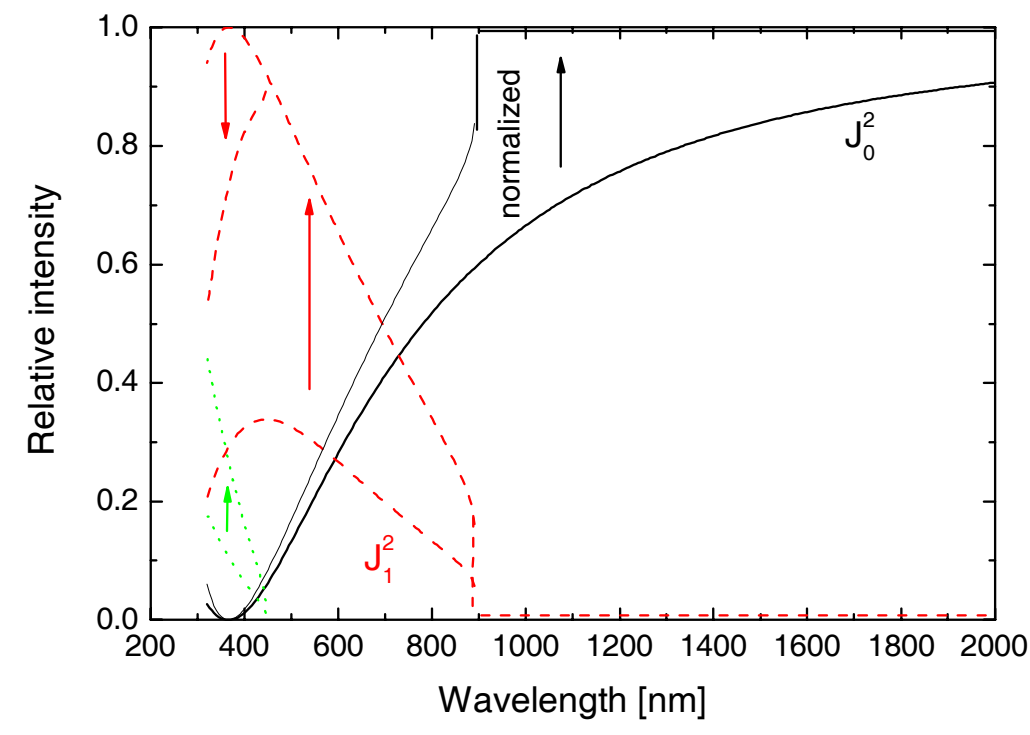

Figure 3: Intensities of zero (full lines), first order (dashed lines) and second order (dotted lines) according to eq. (2). The effect of the normalization is illustrated by the arrows. 
Below $890 \mathrm{~nm}$ the conditions for propagation of zero and first order diffraction are met. At the onset, eq. (2) predicts an intensity of approximately $10 \%$ which is distributed into each of the two propagating first order modes. Using the normalization, this gives rise to a sudden drop in the specular beam intensity. This phenomenon of sudden changes is known as grating anomaly. Note, that the effect of the grating anomaly is not clearly visible in the measured data shown in Figure 2 because it is superimposed with an absorption effect due to the excitation of a surface plasmon resonance.

In the range between 500 and $800 \mathrm{~nm}$ the diffuse reflection is equivalent to the intensity diffracted into first order. The behavior of diffuse and specular reflection is well explained after the application of the normalization procedure. At lower wavelengths the specular beam shows a minimum because $\mathbf{J}_{0}$ becomes zero for an argument 2.40 . Taking the grating amplitude of $70 \mathrm{~nm}$, we expect the suppression of the zero order beam at a wavelength of $370 \mathrm{~nm}$. In the region from 300 to $445 \mathrm{~nm}$ the normalization has to be adopted for the appearance of second order diffraction. For each case, the effect of the normalization is illustrated by the arrows in Figure 3. Note that the diffuse reflection in the second order regime does not resolve the individual contributions of first and second order diffraction but only their sum.

For assessing the propagation inside of the solar cell structure, we have to consider the refractive index of the absorber layer which is illustrated in Figure 4. Desirable would be the case of diffraction into high angles for the spectral range between 650 and $800 \mathrm{~nm}$ (amorphous cells) and 700 to $1100 \mathrm{~nm}$ (microcrystalline cells). For the shown grating with a period of 890 $\mathrm{nm}$ this would be the case for diffraction into fourth or possibly fifth order.

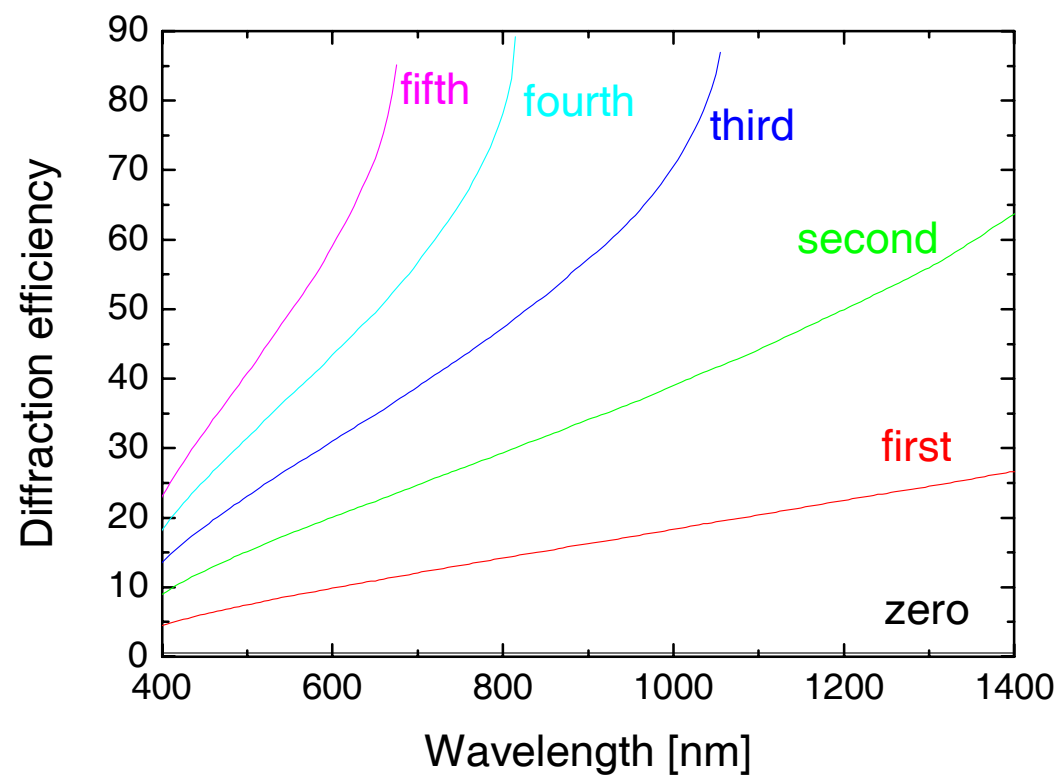

Figure 4: Diffraction angles assuming the refractive index of silicon, the horizontal scale refers to the wavelength in vacuum.

Figure 5 illustrates the results of the normalization procedure for the case of propagation in silicon. It shows that the contributions of fourth and fifth order are quite moderate. Different 
from the behavior of the first order onset shown in Figure 3, their intensities are low around their onset. In the wavelength range that is interesting for microcrystalline silicon (800 to1100 nm), we find dominant diffraction into second order, but also considerable zero order intensity. Between 650 and $800 \mathrm{~nm}$, most of the zero order diffraction is suppressed because $\mathrm{J}_{0}$ goes through its second zero at 5.52. The particular dimensions of this grating could thus be interesting for amorphous silicon solar cells.

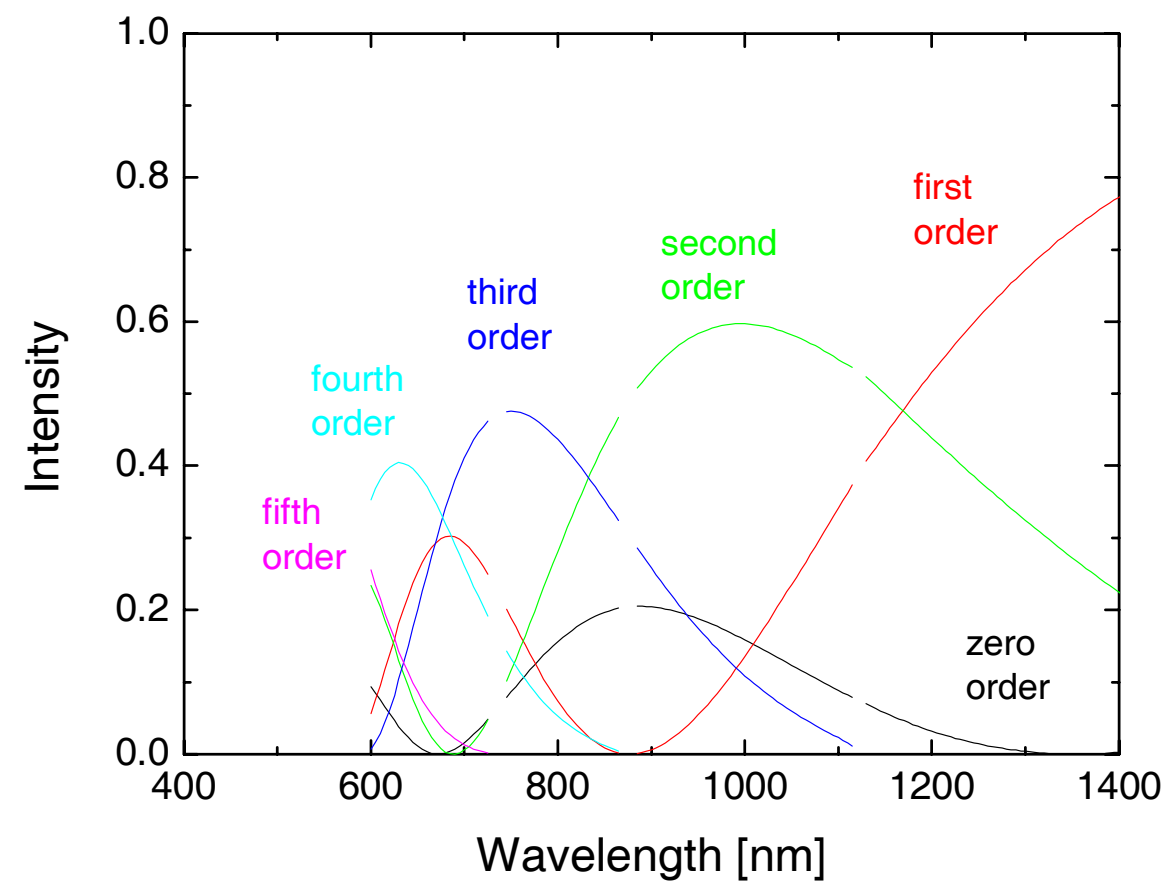

Figure 5: Normalized intensity of the propagating orders for the case of diffraction into amorphous silicon. The horizontal scale again refers to the wavelength in vacuum.

\section{B) Surface plasmon excitation at the metal/dielectric interface}

Figure 2 showed a strong absorption feature at the onset of first order diffraction. We attribute this effect to the excitation of surface plasmon polaritons (SPPs) which are mediated by the texture. This excitation reduces the reflection of the back contact and must therefore be considered as undesired loss mechanism in solar cells.

First observed by Ritchie in 1957 [18], surface plasmon polaritons are propagating waves of the surface charge distribution at the interface between a metal and a dielectric. The dispersion relation of a SPP on a flat, semi-infinite metal surface is given by the following relation (e.g. [19], eqn. (2.5)):

$$
k(\omega)= \pm \frac{\omega}{c}\left(\frac{\varepsilon_{1} \varepsilon_{2}(\omega)}{\varepsilon_{1}+\varepsilon_{2}(\omega)}\right)^{1 / 2}
$$

In this equation $\varepsilon_{1}$ and $\varepsilon_{2}$ denote the dielectric functions of air or a dielectric, and the metal, respectively. For the sake of simplicity we consider only the real part of the dielectric 
function which is justified for silver in the IR and visible where the magnitude of $\operatorname{Re}\left(\varepsilon_{\mathrm{Ag}}\right)$ exceeds by far that of $\operatorname{Im}\left(\varepsilon_{\mathrm{Ag}}\right)$. Figure 6 shows that the dispersion relation of the SPP never intersects with the dispersion relation of a photon which is simply given by the linear relationship $k(\omega)=\omega / c$. In other words, the conservation of energy and momentum prevents the direct excitation of SPPs by light on flat surfaces. Surface modulations, however, give very effective coupling to radiation [20-22]. Generally, an arbitrary structure offers little control on the excitation of SPPs, but the situation is different for periodically structured surfaces where grating coupling is mediated by multiples of the reciprocal lattice vector $G=2 \pi / L$.

$$
k=k_{0} \sin \theta+m \cdot \frac{2 \pi}{L}
$$

Here, the quantity $k_{0}$ is the wave vector of the photon in vacuum, the factor $\sin \theta$ selects the in-plane part of the photon wave vector, $L$ is the period of the grating, and $m$ is again an integer number. Grating coupling is illustrated in Figure 6. According to their dispersion relation; photons are represented by straight lines; the cases " $\pm 90^{\circ}$ " correspond to the highest possible in-plane momentum of photons that propagate under grazing angles over the surface. Illumination under a different inclination lowers the in-plane wave vector; in the dispersion diagram this corresponds to straight lines with higher slope. For example, the shown points correspond to a measurement under an angle of $7^{\circ}$ which was used in the spectrometer.

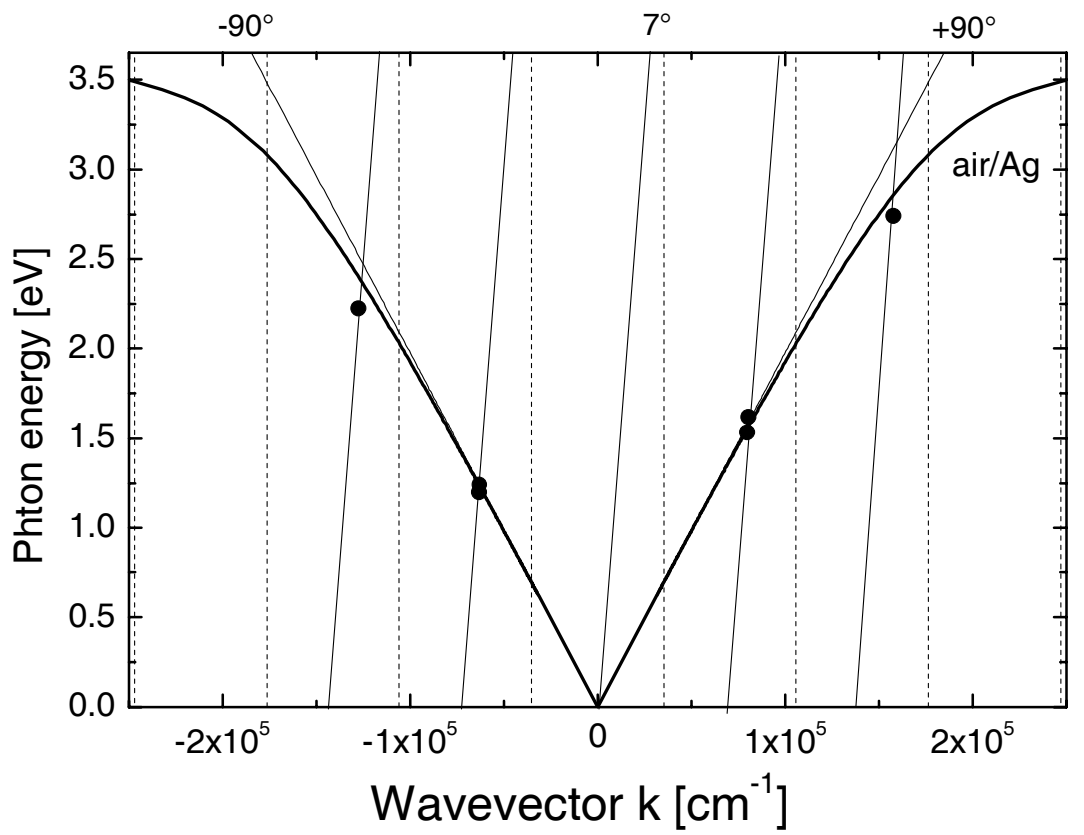

Figure 6: Dispersion relation of the surface plasmon resonance of the flat silver surface (full curves). The presence of a grating allows the definition of Brillouin zones denoted by vertical dashed lines. The periodicity also adds multiples of the reciprocal lattice vector $G$ to the momentum of a photon (angle of incidence of $7^{\circ}$, inclined straight lines). Symbols denote measured dips in reflection.

Grating coupling according to eq (4) shifts the characteristic of the $7^{\circ}$ photon by multiples of the reciprocal lattice vector, and the lowest energy interactions are expected for 1.24 $\mathrm{eV}$ and $1.58 \mathrm{eV}$. Incidentally, the diffractive properties of the grating are also contained in this 
kind of diagram; both intersections with the SPP are just below the crossing points with the grazing photon lines which represent the appearance of the -1 and +1 order at $90^{\circ}$ against the surface normal, respectively. Note that it was not possible to resolve the $1^{\text {st }}$ order grating anomaly in Figure 2, simply because it is hidden in the reflection dip of the plasmon absorption which is close by.

Figure 6 shows that the positions of the reflection dips are in good agreement with the theoretical curve. A close-up reveals slight differences because the grating represents a perturbation from the case of the flat surface. Nevertheless, taking the known geometry as input, we can use this kind of measurement as tool for sampling of the dispersion relation at well defined values of the wave vector. Figure 7 shows such measurements on bilayer samples consisting of $\mathrm{ZnO} / \mathrm{Ag}$ on the grating. With increasing thickness of the $\mathrm{ZnO}$ layer, the measured values of the bi-layer samples approach the theoretical curve of the semi-infinite $\mathrm{ZnO} / \mathrm{Ag}$ interface. In fact, a thin layer of $60 \mathrm{~nm}$ of $\mathrm{ZnO}$ acts almost like bulk material for energies higher than $2 \mathrm{eV}$.

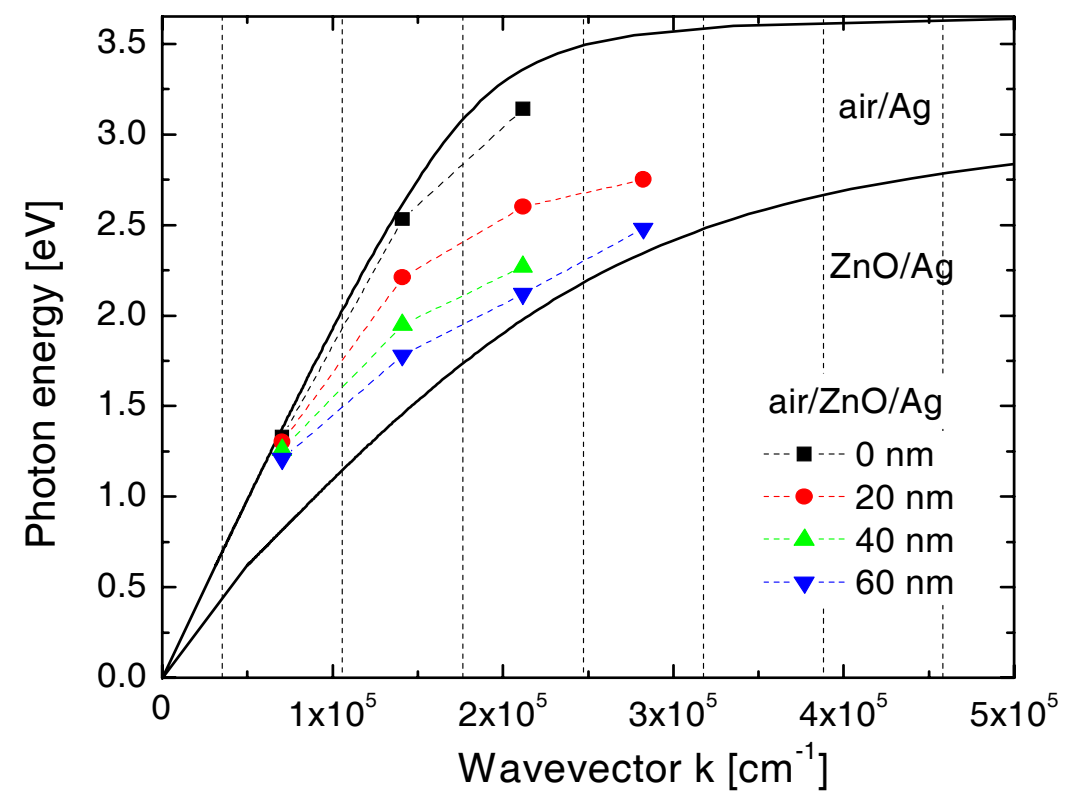

Figure 7: Measurements on silver after coating with thin films of $\mathrm{ZnO}$. The symmetry allows collapsing into the first quadrant, additionally the photon incidence is changed to perpendicular $\left(0^{\circ}\right.$ with respect to the surface normal).

In the high energy part of the diagram the curves tend to flatten out. Due to the finite width of the absorption in terms of energy (c.f. Figure 2), it becomes difficult to uniquely resolve individual dips at higher energies than those shown. We think that this flat part of the dispersion relations forms a link between our findings on periodic gratings and observations on arbitrary structures; even for random textures, excitation in this region becomes possible due to the fact that all wave vectors have almost the same energy. Energy-momentum conservation is thus relaxed. In other words, coupling can proceed for all sorts of texture, random and periodic alike [23]. For the theoretical dispersion relations in Figure 6 we can easily determine the plateau energies by demanding the denominator of eq. (3) to become zero. We find 3.6 and $3.0 \mathrm{eV}$ for 
the interfaces of $\mathrm{Ag}$ with air and $\mathrm{ZnO}$, respectively. The measured data for the air/ $\mathrm{Ag}$ interface suggest somewhat lower energies due to deviations from the ideal case of the flat surface.

For reflection measurements on randomly textured surfaces, Springer reports surface plasmon resonance absorption at 3.5 and $2.8 \mathrm{eV}$ for the air/silver and the $\mathrm{ZnO} /$ silver interfaces, respectively [24]. In ellipsometric studies of rough $\mathrm{ZnO} / \mathrm{Ag}$ interfaces Sainju reports a Lorentzian resonance whose peak energy decreases from 3 to $2.75 \mathrm{eV}$ for increasing surface roughness [25]. The limiting values of our measurements appear to correspond well with the reported data.

In addition, we are able to map the dispersion relation of the $\mathrm{ZnO} / \mathrm{Ag}$ interface also for lower energies. We think that this information could also be important for random textures because the dispersion relations should be very similar as long as the rms roughness is moderate and can be considered a perturbation of the flat surface. In the absence of a well defined periodicity, we expect that plasmon absorption is mediated by a distribution of length scales that is centered about the correlation length. We could then try to estimate the energy range where the excitation of plasmon resonances may be expected from the experimentally determined dispersion relations shown in Figure 7.

\section{CONCLUSIONS}

We discussed the effect of using periodic textures as diffusing elements in solar cells. For a sinusoidal model structure we were able to predict absorption enhancement in silicon films due to diffraction phenomena. Our ongoing work on textured back reflectors revealed conditions where the excitation of surface plasmon resonances can substantially lower the performance of the back reflector. Furthermore, we were able to use grating coupled plasmon resonances for mapping the dispersion relation of $\mathrm{ZnO} / \mathrm{Ag}$ back reflectors. We find that this is a simple method that gives access to a wide spectral range, and we think that this information is also useful for arbitrary textures.

\section{ACKNOWLEDGEMENTS}

We thankfully acknowledge funding within the European Project "Flexcellence" under contract 019948, and by the Swiss Commission for Technology and Innovation (CTI) under contract 8809.2.

\section{REFERENCES}

[1] D. E. Carlson and C. R. Wronski, Applied Physics Letters 28 (1976) 671.

[2] H. W. Deckman, C. R. Wronski, H. Witzke, and E. Yablonovitch, Applied Physics Letters 42 (1983) 968.

[3] M. S. Bennett, J. L. Newton, and K. Rajan, in Proc. 7th European PVSEC, Sevilla, 1986, p. 544.

[4] J. Meier, J. Spitznagel, S. Faÿ, C. Bucher, U. Graf, U. Kroll, S. Dubail, and A. Shah, in Proc. 29th IEEE PVSC, New Orleans, 2002, p. 1118. 
[5] M. Kambe, M. Fukawa, N. Taneda, Y. Yoshikawa, K. Sato, K. Ohki, S. Hiza, A. Yamada, and M. Konagai, in Proc. 3rd World PVSEC, Osaka, 2003.

[6] J. Müller, B. Rech, J. Springer, and M. Vanecek, Solar Energy 77 (2004) 917.

[7] A. Banerjee and S. Guha, Journal of Applied Physics 69 (1991) 1030.

[8] S. K. Hayashi, K.; Yamamoto, K., Solid state communications 79 (1991) 763.

[9] M. Westphalen, U. Kreibig, J. Rostalski, H. Lüth, and D. Meissner, Solar Energy Materials \& Solar Cells 61 (2000) 97.

[10] B. P. Rand, P. Peumans, and S. R. Forrest, Journal of Applied Physics 96 (2004) 7519.

[11] E. Moulin, P. Luo, J. Sukmanowski, M. Schulte, F. X. Royer, and H. Stiebig, in Proc. 22nd European PVSEC, Milan, 2007, p. 2195.

[12] T. C. Paulick, Journal of Applied Physics 62 (1987) 3016.

[13] C. Heine and R. H. Morf, Applied Optics 34 (1995) 2476.

[14] C. Eisele, C. E. Nebel, and M. Stutzmann, Journal of Applied Physics 89 (2001) 7722.

[15] F.-J. Haug, T. Söderström, M. Python, V. Terrazzoni-Daudrix, X. Niquille, and C. Ballif, To be published in Sol. En. Mat. (2008)

[16] P. Beckmann and A. Spizzichino, The scattering of electromagnetic waves from rough surfaces, Artech House, Inc., Norwood (MA) 1987.

[17] J. E. Harvey and A. Krywonos, in Proc. SPIE AM100-26, Denver, 2004.

[18] R. H. Ritchie, Physical Review 106 (1957) 874.

[19] H. Raether, Surface Plasmons, Springer Berlin, 1988.

[20] R. H. Ritchie, E. T. Arakawa, J. J. Cowan, and R. N. Hamm, Physical Review Letters 21 (1968) 1530.

[21] B. Laks, D. L. Mills, and A. A. Maradudin, Physical Review B 23 (1981) 4965.

[22] N. E. Glass, M. Weber, and D. L. Mills, Physical Review B 29 (1984) 6548.

[23] E. Kretschmann, T. L. Ferrell, and J. C. Ashley, Physical Review Letters 42 (1979) 1312.

[24] J. Springer, B. Rech, W. Reetz, J. Muller, and M. Vanecek, Solar Energy Materials and Solar Cells 85 (2005) 1.

[25] D. Sainju, P. J. van den Oever, N. J. Podraza, M. Syed, J. A. Stoke, J. Chen, X. Yang, X. Deng, and R. W. Collins, in Proc. 4th World PVSEC, Hawaii, 2006. 